\title{
Prior appendectomy and cerebral infarction as potential risk factors for recurrent ischemic colitis: a case control study
}

Takuma Higurashi ( $\nabla$ takuma_h@yokohama-cu.ac.jp )

Yokohama City University Graduate School of Medicine

Tomohiro Takatsu

Yokohama City University Graduate School of Medicine

Noboru Misawa

Yokohama City University Graduate School of Medicine

Tsutomu Yoshihara

Yokohama City University Graduate School of Medicine

Keiichi Ashikari

Yokohama City University Graduate School of Medicine

Takaomi Kessoku

Yokohama City University Graduate School of Medicine

Hidenori Ohkubo

Yokohama City University Graduate School of Medicine

Kunihiro Hosono

Yokohama City University Graduate School of Medicine

Masato Yoneda

Yokohama City University Graduate School of Medicine

Satoru Saito

Yokohama City University Graduate School of Medicine

Atsushi Nakajima

Yokohama City University Graduate School of Medicine

\section{Research Article}

Keywords: acute lower gastrointestinal bleeding, ischemic colitis, appendectomy

Posted Date: February 2nd, 2022

DOI: https://doi.org/10.21203/rs.3.rs-1253104/v1 
License: (c) (i) This work is licensed under a Creative Commons Attribution 4.0 International License. Read Full License 


\section{Abstract}

Background: Ischemic colitis (IC) is a benign disease associated with acute lower gastrointestinal bleeding and frequent recurrence. While several studies have investigated risk factors for IC onset, few have assessed the risk factors for recurrent IC. This study aimed to identify risk factors for recurrent IC.

Methods: Potential risk factors for recurrence were assessed by examining medical records and laboratory findings in this single-center retrospective study. We extracted the following data from the patients' medical records: patient characteristics, clinical signs and symptoms, laboratory findings, method of treatment, length of hospital stay, disease course, and the frequency of IC morbidities. Patients with IC were selected from a total of 439,312 patients over an 11-year period. Patients were divided into recurrent and non-recurrent IC groups.

Results: In total, 225 patients met the diagnostic criteria for IC during the specified study period; of these, 204 (90.7\%) and 21 (9.3\%) were included in the non-recurrent and recurrent IC groups, respectively. Univariate and multivariate analyses showed a significant association between IC recurrence and both cerebral infarction $(p=0.01$, odds ratio $=5.41)$ and history of appendectomy $(p=0.0009$, odds ratio $=$ 5.19). The median (interquartile range [IQR]) follow-up time for all patients was 1556 (353-2768) days; this was much longer than the median (IQR) time to recurrence of 291 (64-907) days in the recurrent IC group.

Conclusions: Prior cerebral infarction and appendectomy are potential risk factors for IC recurrence. However, the pathogenic mechanism of IC remains to be determined.

Trial registration: B200400003 (Yokohama City University Certified Institutional Review Board, March 27th, 2020), UMIN ID 000044303 (retrospectively registered, May 24th, 2021)

\section{Background}

Ischemic colitis (IC) is one of the most common causes of acute lower gastrointestinal bleeding, and its diagnostic criteria were proposed by Marston et al. in 1966 (1). IC accounts for $8.7 \%-18.0 \%$ of all cases of acute lower gastrointestinal bleeding (2-4), and according to a previous systematic review, its incidence in the general population ranges from 4.5 to 44 cases per 100,000 person-years (5). IC is generally more common in older women (6-14) and is characterized by a sudden onset of left lower quadrant pain accompanied by diarrhea and bloody stools. While these symptoms often rapidly resolve with bowel rest, some cases involve irreversible changes that require surgery (e.g., gangrenous colitis, fulminant colitis, stricture formation, and chronic IC) or may be fatal. IC may also be caused by other severe conditions. For example, systemic hypotension due to sepsis, hypokalemia, cardiogenic shock, or third-space fluid shift causing intestinal hypoperfusion, thereby progressing to IC. Vascular (e.g., hypertension, dyslipidemia, diabetes, cardiovascular disease, cerebral infarction, and chronic renal failure) and intestinal (e.g., irritable bowel syndrome, constipation, colorectal cancer, history of abdominal surgery, and history of vascular surgery) factors have been reported to be associated with IC onset (6-18). In recent years, the use of 
drugs, such as contraceptives and vasoconstrictors, has been reported to be a risk factor for IC onset (17, 19, 20). In addition, diseases such as systemic lupus erythematosus and antiphospholipid syndrome, which cause vasculitis, have also been reported as risk factors for IC $(6,21-23)$. However, as these diseases can also cause enteritis, care must be taken in formulating the differential diagnosis of IC.

IC reportedly recurs at a frequency of $6.8 \%-16.0 \%$ after symptom resolution (24-27). While several studies have assessed the risk factors for IC onset, few have investigated the potential risk factors for recurrent IC. A previous study reported smoking and abdominal aortic aneurysms (AAAs) as risk factors for recurrent IC; however, its sample size was relatively small (118 patients) (24).

A recent audit of patients treated for IC at our hospital revealed the absence of AAAs. However, many patients had a history of appendectomy. Therefore, we conducted a retrospective study of patients diagnosed with IC at our hospital and investigated the potential risk factors for recurrent IC.

\section{Methods}

\section{Study design and setting}

The study protocol was approved by the appropriate ethics committee (Yokohama City University Certified Institutional Review Board) (Approval No: CRB3180007) and was exempted from obtaining consent directly from patients by using an opt-out method. We conducted a case-control study of patients previously diagnosed with IC at our hospital during an 11-year interval between January 1, 2009, and December 31, 2019. Patients diagnosed with IC were selected from a total of 439,312 patients registered in the electronic medical record database during this period. The follow-up period was from the onset of IC to the date of the last visit (up to December 31, 2019). Patients who had a relapse after resolution of the initial IC episode during the follow-up period were included in the recurrent IC group, whereas those who experienced a single episode of IC during the study period were included in the nonrecurrent IC group.

\section{Patient characteristics}

We identified patients with IC in the database using the following International Classification of Diseases10 codes: K550 (NDNL, GK7L, PR7K), K551 (F1A5, BPJQ, G440), and K559. We counted cases with multiple IC disease classifications as a single case, and a total of 316 patients with IC were identified. The diagnosis of IC was further confirmed based on clinical symptoms and laboratory findings, as there are no internationally standardized diagnostic criteria for this disease. We used the diagnostic criteria proposed by Marston et al., (1) which are commonly followed in Japan: 1) sudden-onset left lower abdominal pain accompanied by diarrhea and bloody stools, 2) typical findings on contrast-enhanced computed tomography (CT) (edematous thickening of the intestinal wall and increased peri-intestinal fatty tissue concentration) or on colonoscopy (CS) (ulceration/erosion, redness, edema, biopsy image), predominantly on the left side of the colon, 3) no autoimmune disease or infection, 4) no trauma or 
mechanical factors (e.g., hernia), 5) no abdominal surgery within the past 6 months, 6) no evidence of severe acute ischemic changes in other organs, and 7) absence of a secondary onset of IC. We excluded patients who were younger than 20 years, pregnant, or opted out of the study; we also excluded suspected cases that could not be definitively diagnosed, and patients diagnosed with IC at other hospitals, as it was not possible to confirm what diagnostic criteria were used.

\section{Data extraction}

We extracted the following data from the patients' medical records: patient characteristics (sex, age, body mass index [BMI], drinking history, smoking history, medical history, comorbidities, medications, and vital signs), clinical signs and symptoms (abdominal pain, diarrhea, bloody stools, and peritoneal irritation symptoms), laboratory findings (contrast-enhanced CT findings, endoscopic features, and histological features), method of treatment, length of hospital stay, disease course (time of resolution of abdominal pain, time of resolution of bloody stools, and initiation of meals), and the frequency of IC morbidities. Contrast-enhanced CT and/or CS was performed to assess each segment of the colon. Contrastenhanced CT was performed to detect poor contrast, bowel wall thickening, mesenteric fat stranding, pericolonic free fluid, mesenteric gas, and portal vein gas. CS was performed to assess the presence of mucosal erosion, ulceration, edema, redness, necrosis, and stricture. The frequency of IC morbidities was defined as the number of times the patient was diagnosed with IC at our hospital.

\section{Statistical analysis}

All patient data were entered into a Microsoft Excel 2016 (Microsoft Corporation, Redmond, WA, USA) spreadsheet. The computer containing the data was password-protected and stored in a locked cabinet in the medical office. Continuous variables are expressed as mean \pm standard deviation, and categorical variables are expressed as numbers and percentages. The chi-square test was performed to compare proportions between the recurrent IC and non-recurrent IC groups, and the t-test was used to compare means. All statistical analyses were performed using JMP Pro 15 (SAS, Cary, NC, USA), and the level of statistical significance $(p)$ was set at $\leq 0.05$. Two separate multivariate analyses were performed including 1) three items that were previously reported to be significantly different (current smoking (24) and two items that were significantly different in the univariate analyses) and 2) all items. These three items were also analyzed for the time to IC recurrence using the Kaplan-Meier method.

\section{Results}

Of the 316 patients diagnosed with IC from the medical records database, 91 were excluded based on our diagnostic criteria; therefore, we finally included the data of 225 patients in our analyses. The recurrent and non-recurrent IC groups comprised 21 (9.3\%) and 204 (90.7\%) patients, respectively (Figure 1). 


\section{Characteristics of patients with IC}

The mean age of the patients was $64.6 \pm 15.0$ years and most were female $(70.22 \%)$ (Table 1$)$. BMI and drinking/smoking history data were only available for 178 (79.1\%) and 203 (89.8\%) patients, respectively, because of the inclusion of emergency patients in addition to general internal medicine outpatients. Smoking history was a risk factor for IC, and 129 (57.3\%) out of the 225 patients were non-smokers. While abdominal pain was the first symptom in all patients, bloody stool was not the first symptom in any patient. Hospitalization was required for $44.4 \%$ of the patients, and the mean ( \pm SD) duration of hospitalization was $8.9( \pm 6.3)$ days. There was no significant difference in the length of hospitalization between the recurrent and non-recurrent IC groups, and there was no mortality among either inpatients or outpatients. The medical records documented the entire disease course (i.e., time from IC onset to the resolution of symptoms and initiation of dietary therapy) for hospitalized patients. The time (from IC onset) to resolution of bloody stools and abdominal pain, as well as the resumption of oral intake, were $3.6( \pm 1.4), 4.9( \pm 1.8)$, and $5.8( \pm 2.5)$ days $( \pm S D)$, respectively. No significant differences were observed between the recurrent and non-recurrent IC groups in this regard.

Table 1

Patient characteristics 


\begin{tabular}{|c|c|}
\hline Recurrent and non-recurrent IC & $\mathrm{n}(\%)$ \\
\hline Number of cases & 225 \\
\hline Age $($ mean \pm SD) & $64.6 \pm 15$ \\
\hline \multicolumn{2}{|l|}{ Sex } \\
\hline Male & $67(29.8)$ \\
\hline Female & $158(70.2)$ \\
\hline \multicolumn{2}{|l|}{$\mathrm{BMI}\left[\mathrm{kg} / \mathrm{m}^{2}\right]$} \\
\hline Mean \pm SD & $22.2 \pm 3.8$ \\
\hline $\mathrm{BMI}<18.5$ & $23(12.9)$ \\
\hline $18.5 \leq \mathrm{BMI}<25$ & $125(70.2)$ \\
\hline $\mathrm{BMI} \geq 25$ & $30(16.9)$ \\
\hline \multicolumn{2}{|l|}{ Alcohol } \\
\hline Non-drinker & $99(44.0)$ \\
\hline Ethanol $<60 \mathrm{~g} /$ day & $88(39.1)$ \\
\hline $60 \mathrm{~g} /$ day $\leq$ Ethanol $<100 \mathrm{~g} /$ day & $14(6.2)$ \\
\hline Ethanol $\geq 100 \mathrm{~g} /$ day & $1(0.4)$ \\
\hline No data & $23(10.2)$ \\
\hline \multicolumn{2}{|l|}{ Smoking } \\
\hline Non-smoker & $129(57.3)$ \\
\hline Past smoker & $44(19.6)$ \\
\hline Current smoker & $29(12.9)$ \\
\hline No data & $23(10.2)$ \\
\hline Hospitalization & $100(44.4)$ \\
\hline Period (Mean \pm SD) (days) & $8.9 \pm 6.3$ \\
\hline Time until bloody stools disappear (days) & $3.6 \pm 1.4$ \\
\hline Time until abdominal pain disappears (days) & $4.9 \pm 1.8$ \\
\hline Time to start of oral intake (days) & $5.8 \pm 2.5$ \\
\hline \multicolumn{2}{|l|}{ Medicines } \\
\hline PPI & $60(26.7)$ \\
\hline
\end{tabular}




\begin{tabular}{|c|c|}
\hline $\mathrm{H}_{2}$ blockers & $14(6.2)$ \\
\hline Steroid & $16(7.1)$ \\
\hline Immunosuppressants & $10(4.4)$ \\
\hline Antibiotics & $8(3.6)$ \\
\hline Contraceptives & $0(0)$ \\
\hline NSAIDs & $14(6.2)$ \\
\hline Hormone pills & $12(5.3)$ \\
\hline Laxatives & $26(11.6)$ \\
\hline Stomach and bowel medications & $21(9.3)$ \\
\hline Antidiarrheals & $1(0.44)$ \\
\hline Antiplatelets & $36(16)$ \\
\hline Anticoagulants & $11(4.9)$ \\
\hline Other antithrombotic & $8(3.6)$ \\
\hline \multicolumn{2}{|l|}{ Medical history and comorbidities } \\
\hline Hypertension & $113(50.2)$ \\
\hline Dyslipidemia & $79(35.1)$ \\
\hline Diabetes & $29(12.9)$ \\
\hline Cardiovascular disease & $47(20.9)$ \\
\hline Atrial tachycardia & $13(5.8)$ \\
\hline Heart disease & $28(12.4)$ \\
\hline Vessels beyond the heart & $10(4.4)$ \\
\hline Liver diseases & $25(11.1)$ \\
\hline Intestinal diseases & $57(25.3)$ \\
\hline Colon cancer & $6(2.7)$ \\
\hline Rectal cancer & $2(0.9)$ \\
\hline Diverticulitis & $2(0.9)$ \\
\hline Diverticular hemorrhage & $2(0.9)$ \\
\hline Irritable bowel syndrome & $4(1.8)$ \\
\hline lleus & $6(2.7)$ \\
\hline
\end{tabular}


Cerebral infarction

Cerebral hemorrhage

Endocrine disorders

Hematological disease

Collagen disease

Psychiatric disorder

Hemodialysis

Total abdominal surgeries

Appendectomy

Gynecological surgery

Abdominal surgery other than the above

Gastric surgery

Small bowel surgery

Colon surgery

Rectal surgery

Liver surgery

Gallbladder surgery

Pancreatic surgery

Abdominal hernia

Malignant disease

Colorectal cancer

Chemotherapy
$15(6.7)$

8 (3.7)

15 (6.7)

7 (3.1)

13 (5.8)

20 (8.9)

6 (2.7)

93 (41.3)

44 (19.6)

46 (20.4)

25 (11.1)

6 (2.7)

3 (1.3)

5 (2.2)

2 (0.9)

4 (1.8)

8 (3.6)

4 (1.8)

2 (0.9)

51 (22.7)

7 (3.1)

19 (8.4)

BMI, body mass index; $\mathrm{H} 2$ blocker, histamine $\mathrm{H} 2$-receptor antagonist; IC, ischemic colitis; NSAIDs, nonsteroidal anti-inflammatory drugs; PPI, proton pump inhibitor; SD, standard deviation

The distribution of comorbidities that were considered vascular risk factors for the development of IC was as follows: hypertension $(n=113,50.22 \%)$, dyslipidemia $(n=79,35.11 \%)$, diabetes $(n=29,12.89 \%)$, cardiovascular disease $(n=38,16.89 \%)$, cerebral infarction $(n=15,6.67 \%)$, and chronic renal failure with dialysis $(n=6,2.67 \%)$. Intestinal factors included constipation $(n=41,18.22 \%)$, colorectal cancer $(n=7$, $3.11 \%)$, and abdominal surgery $(n=93,41.33 \%)$. Cases of intestinal obstruction or ischemia secondary to severe strictures caused by colorectal cancer were excluded. 


\section{Comparison of risk factors between the study groups}

Two patients in the recurrent IC group experienced a second episode of IC, but no patients had more than three recurrences. Most risk factors for IC development were not significantly different between the recurrent and non-recurrent IC groups (Table 2); these included current smoking, which was previously reported as a risk factor for recurrence. No cases of AAA were observed. Cerebral infarction $(p=0.0128$, odds ratio $[\mathrm{OR}]=5.41)$ and appendectomy $(p=0.0009$, $\mathrm{OR}=5.19)$ were the only comorbidities significantly associated with IC recurrence in the univariate analyses and both multivariate analyses (Table 3, Figure 2, Figure 3, Figure 4).

\section{Table 2}

Univariate analysis of risk factors for IC. 


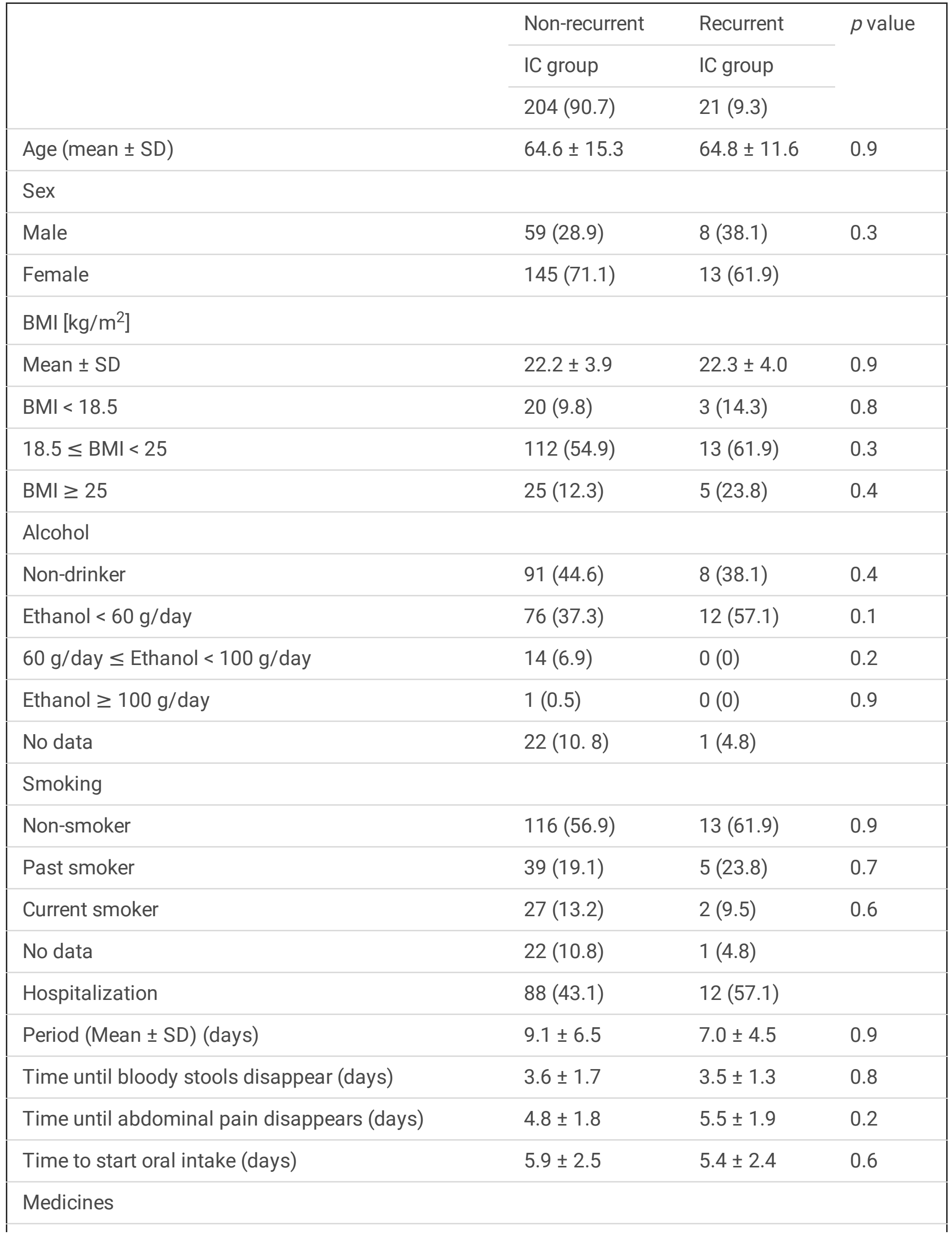




\begin{tabular}{|c|c|c|c|}
\hline PPIs & $52(25.5)$ & $8(38.1)$ & 0.2 \\
\hline H2 blockers & $13(6.4)$ & $1(4.8)$ & 1 \\
\hline Steroids & $16(7.8)$ & $0(0)$ & 0.4 \\
\hline Immunosuppressants & $10(4.9)$ & $0(0)$ & 0.6 \\
\hline Antibiotics & $8(3.9)$ & $0(0)$ & 1 \\
\hline Contraceptives & $0(0)$ & $0(0)$ & \\
\hline NSAIDs & $11(5.4)$ & $3(14.3)$ & 0.1 \\
\hline Hormone pills & $11(5.4)$ & $1(4.8)$ & 1 \\
\hline Laxatives & $21(10.3)$ & $5(23.8)$ & 0.08 \\
\hline Stomach and bowel medications & $19(9.3)$ & $2(9.5)$ & 1 \\
\hline Antidiarrheals & $1(0.5)$ & $0(0)$ & 1 \\
\hline Antiplatelets & $31(15.2)$ & $5(23.8)$ & 0.3 \\
\hline Anticoagulants & $11(5.4)$ & $0(0)$ & 0.6 \\
\hline Other antithrombotics & $7(3.4)$ & $1(4.8)$ & 0.5 \\
\hline \multicolumn{4}{|l|}{ Medical history } \\
\hline Hypertension & $100(49.0)$ & $13(61.9)$ & 0.3 \\
\hline Dyslipidemia & $69(33.8)$ & $10(47.6)$ & 0.2 \\
\hline Diabetes & $25(12.1)$ & $4(19.1)$ & 0.3 \\
\hline Cardiovascular disease & $40(19.6)$ & 7 (33.3) & 0.1 \\
\hline Arrhythmia & $11(5.4)$ & $2(9.5)$ & 0.3 \\
\hline Heart disease & $25(12.3)$ & $3(14.3)$ & 0.7 \\
\hline Vessels beyond the heart & $8(3.9)$ & $2(9.5)$ & 0.2 \\
\hline Liver disease & $21(10.3)$ & $4(19.1)$ & 0.3 \\
\hline Intestinal disorders & $50(24.5)$ & 7 (33.3) & 0.4 \\
\hline Colon cancer & $5(2.5)$ & $1(4.8)$ & 0.4 \\
\hline Rectal cancer & $2(1)$ & $0(0)$ & 1 \\
\hline Diverticulitis & $2(1)$ & $0(0)$ & 1 \\
\hline Diverticular hemorrhage & $1(0.5)$ & $1(4.8)$ & 0.2 \\
\hline Irritable bowel syndrome & $3(1.5)$ & $1(4.8)$ & 0.3 \\
\hline
\end{tabular}




\begin{tabular}{|c|c|c|c|}
\hline Ileus & $6(2.9)$ & $0(0)$ & 1 \\
\hline Constipation & $36(17.7)$ & $5(23.8)$ & 0.6 \\
\hline Cerebral infarction & $11(5.4)$ & $4(19.1)$ & 0.04 \\
\hline Cerebral hemorrhage & $7(3.4)$ & $1(4.8)$ & 1 \\
\hline Endocrine disorders & $15(7.4)$ & $0(0)$ & 0.3 \\
\hline Hematological disease & $6(2.9)$ & $1(4.8)$ & 0.5 \\
\hline Collagen disease & $12(5.88)$ & $1(4.76)$ & 1 \\
\hline Psychiatric disorder & $17(8.3)$ & $3(14.3)$ & 0.4 \\
\hline Hemodialysis & $5(2.5)$ & $1(4.8)$ & 0.5 \\
\hline Total abdominal surgeries & $81(39.7)$ & $12(57.1)$ & 0.1 \\
\hline Appendectomy & $34(16.7)$ & $10(47.6)$ & 0.0007 \\
\hline Gynecological surgery & $43(21.1)$ & $3(14.3)$ & 0.6 \\
\hline Abdominal surgery other than the above & $22(10.8)$ & $3(14.3)$ & 0.7 \\
\hline Gastric surgery & $5(2.5)$ & $1(4.8)$ & 0.4 \\
\hline Small bowel surgery & $3(1.5)$ & $0(0)$ & 1 \\
\hline Colon surgery & $4(2.0)$ & $1(4.8)$ & 0.4 \\
\hline Rectal surgery & $2(1.0)$ & $0(0)$ & 1 \\
\hline Liver surgery & $4(2.0)$ & $0(0)$ & 1 \\
\hline Gallbladder surgery & $7(3.4)$ & $1(4.8)$ & 0.5 \\
\hline Pancreatic surgery & $4(2.0)$ & $0(0)$ & 1 \\
\hline Abdominal hernia & $2(1.0)$ & $0(0)$ & 1 \\
\hline Malignant disease & $45(22.1)$ & $6(28.6)$ & 0.3 \\
\hline Colorectal cancer & $6(2.9)$ & $1(4.8)$ & 0.6 \\
\hline Chemotherapy & $15(7.4)$ & $4(19.1)$ & 0.09 \\
\hline
\end{tabular}

Table 3

Multivariate analysis of risk factors for IC. 


\begin{tabular}{|lll|}
\hline & $p$-value & OR \\
\hline Current smoker & 0.2 & $0.4(0.06-2.0)$ \\
\hline Cerebral infarction & $\mathbf{0 . 0 0 8}$ & $6.3(1.6-24.4)$ \\
\hline Appendectomy & $\mathbf{0 . 0 0 0 5}$ & $6.2(2.2-17.4)$ \\
\hline \multicolumn{2}{|l}{ IC, ischemic colitis; OR, odds ratio } \\
\hline
\end{tabular}

The median (interquartile range (IQR)) follow-up time for all patients was 1556 (353-2768) days, and the median (IQR) time to IC recurrence was 291 (64-907) days (Figure 5). The follow-up duration was defined as the period from the onset of IC until the last day of treatment (documented up to December 31, 2019 , in the electronic medical records) for patients whose follow up ended and for those who were discharged or referred to other hospitals during the follow-up period.

\section{Comparison of imaging results between the study groups}

CT was performed in 218 out of the 225 patients within 5 days after IC onset. The findings indicative of IC were most commonly observed in the descending colon, followed by the sigmoid colon and transverse colon; only two patients did not exhibit signs of IC in the descending colon. Edematous thickening of the intestine was observed in all patients, and more than $90 \%$ of patients had disproportionate fat stranding around the intestine. There was no significant difference in the proportion of positive findings in each colon segment between the non-recurrent and recurrent IC groups (Table 4).

CS was performed in 155 patients between the date of IC onset and 95 days later. CS performed 23 days after IC onset often did not yield findings indicative of IC. In contrast, positive findings for IC were observed in all cases when CS was performed within 22 days of IC onset; in two of these cases, insertion was limited to the descending colon, and deep insertion was deferred because of inflammatory findings or severe pain. Among the patients who underwent CS within 22 days, no significant differences were observed in the proportion of positive findings in each colon segment between the non-recurrent and recurrent IC groups (Table 4).

Table 4

Univariate analysis of computed tomography and colonoscopy findings. 


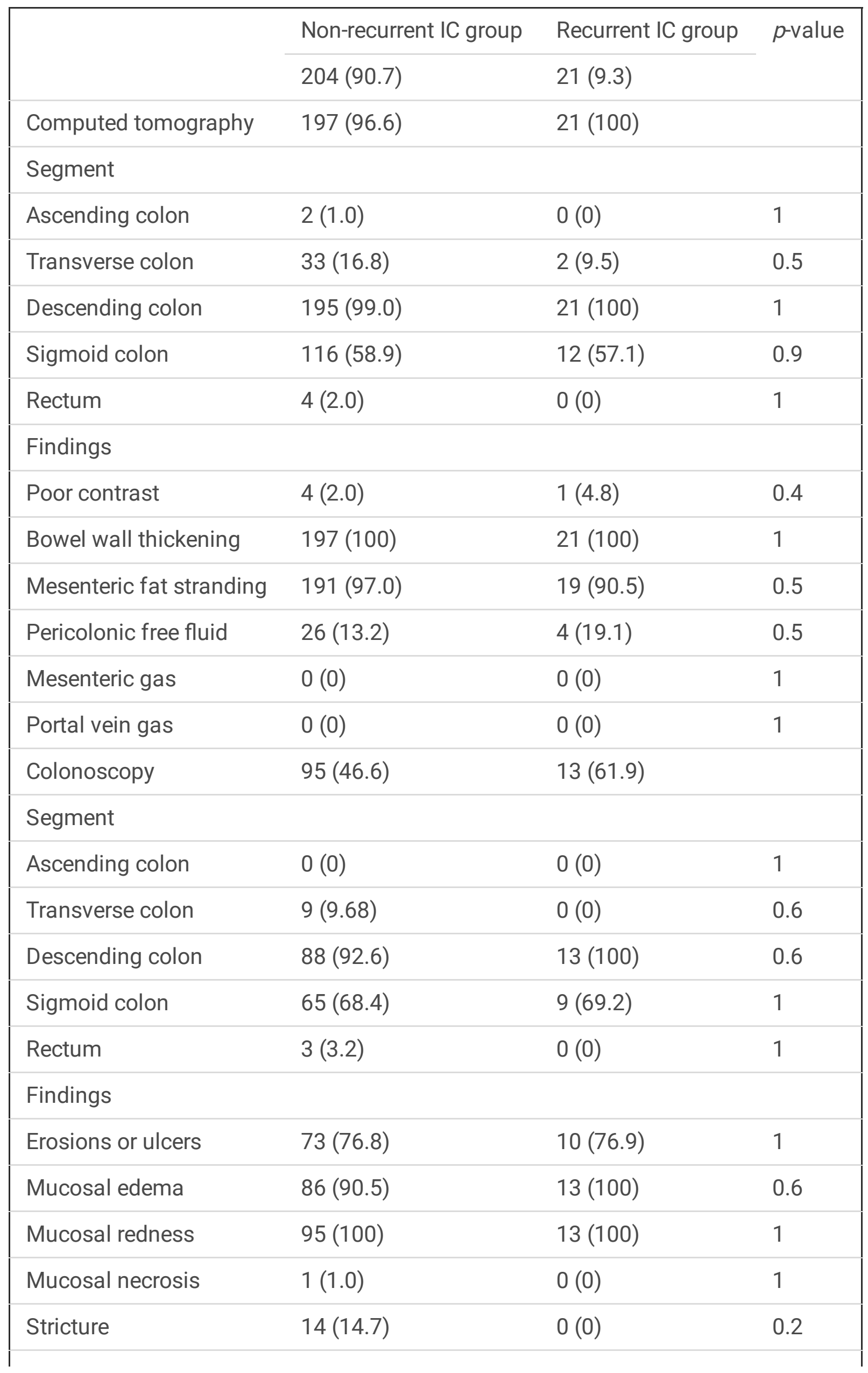




\section{Discussion}

The results of this study indicate that a history of appendectomy or cerebral infarction significantly increased the risk of IC recurrence. In contrast to previous studies, a significant association was not found between IC recurrence and either smoking or AAAs. Prior studies have identified a history of abdominal surgery as a risk factor for IC onset (6-18). However, a history of appendectomy has not been reported as a risk factor for IC onset or recurrence.

The appendix, located in the right lower abdomen and connected to the cecum, is anatomically distant from the site most frequently affected by IC. Recent studies have suggested that the appendix functions as an immune organ $(28,29)$ and that it is associated with intestinal microflora $(30,31)$ and autoimmune bowel diseases (32-34). Ulcerative colitis is an autoimmune bowel disease characterized by lesions extending to the rectum; it has been reported to have a relationship with the appendix, which is anatomically distant. Patients who undergo appendectomy at a young age are less likely to develop ulcerative colitis $(32,33)$. This has been attributed to the fact that appendectomy markedly reduces the proliferative response of lymphocytes in the mucosal intrinsic layer to autologous intestinal bacteria. The appendiceal lymphoid tissue has a vital role in producing immunoglobulin $\mathrm{A}(\lg \mathrm{A})$-positive cells that are mobilized to the colon. Intestinal IgA induced by appendiceal lymphoid tissue contributes to the regulation of the intestinal microbiota. A previous study found that a decrease in the IgA titer greatly disrupted the intestinal microbiota balance in appendectomized mice (30). Therefore, we speculate that disturbances to the intestinal microbiota balance render patients with a history of appendectomy more prone to repeated intestinal inflammation than those without a history of appendectomy.

Cerebral infarction has previously been reported as a risk factor for the development of IC (6-18). Cerebral infarction can be broadly classified as cardiogenic cerebral embolism, lacunar infarction, or atherothrombotic cerebral infarction. Among these, the cause of cardiogenic cerebral embolism is atrial fibrillation, while lacunar infarction and atherothrombotic cerebral infarction are caused by arteriosclerosis. Both atherosclerotic disease and cerebral infarction have been reported to be risk factors for IC onset. In the present study, we did not observe differences in risk factors for atherosclerosis (e.g., smoking, hypertension, obesity, diabetes, and dyslipidemia) between the recurrent and non-recurrent IC groups. One of the reasons may be that the sample size was too small to provide sufficient statistical power to detect a significant difference.

Vascular risk factors for IC are attributed to local microcirculatory disturbances rather than a thromboembolic effect. Therefore, in terms of cerebral infarction, the pathogenic mechanism can be more similar to lacunar and atherothrombotic cerebral infarctions, which are caused by a deterioration of circulatory dynamics due to atherosclerosis, than to cardiogenic cerebral embolism. 
The characteristics of the patients in this study (mean age, $64.6 \pm 15.0$ years; $70.22 \%$ women) were similar to those reported in previous studies (35) and the ACG clinical guideline (16); this supports the generalizability of the results of this study. In addition, although there was some concern that patients in the non-recurrent IC group may have subsequently experienced IC recurrence, this was unlikely as the median (IQR) follow-up time for all patients was 1556 (353-2768) days; this was much longer than the median (IQR) time to recurrence of 291 (64-907) days in the recurrent IC group.

This study has some limitations. First, as this was a single-center study, the distribution of comorbidities may have been biased. Second, the retrospective study design relied on previously registered disease codes from a database; therefore, our results may have been affected by omissions in the medical records and incomplete reporting of medical histories. Third, the multivariate analysis was limited owing to the small number of IC cases relative to the number of investigated risk factors. Fourth, we excluded patients with secondary IC, as the inclusion criteria were based on the diagnostic criteria used in Japan. Thus, we were unable to assess patients with IC and hemodynamic deterioration after surgery for AAAs or during treatment of chronic renal failure, as well as those with stenosis or obstruction caused by colorectal cancer. This may be one of the reasons why the number of patients with AAA was zero and that with chronic renal failure and colorectal cancer was low.

\section{Conclusions}

This study identified cerebral infarction and a history of appendectomy as risk factors for recurrent IC. Previously reported risk factors for IC onset, including hypertension, diabetes mellitus, and dyslipidemia, were not found to be related to IC recurrence in our study. These findings may be used to facilitate the identification of patients at high risk of IC recurrence and the institution of appropriate intervention measures in clinical practice. The results of this study warrant further confirmation by multicenter prospective studies with larger samples sizes.

\section{Abbreviations}

AAAs; abdominal aortic aneurysms

BMl; body mass index

CS; colonoscopy

CT; computed tomography

$\mathrm{H} 2$ blocker; histamine $\mathrm{H} 2$-receptor antagonist

IC; ischemic colitis

NSAIDs; non-steroidal anti-inflammatory drugs 
OR; odds ratio

PPl; proton pump inhibitor

SD; standard deviation

\section{Declarations}

\section{Ethics approval and consent to participate}

The study protocol was approved and exempted from obtaining consent by Yokohama City University Certified Institutional Review Board (Approval No: CRB3180007). The study was conducted in accordance with the ethical standards laid down in an appropriate version of the Declaration of Helsinki (as revised in Brazil 2013).

\section{Consent for publication}

Not Applicable

\section{Availability of data and materials}

The datasets used and/or analyzed during this study are not released to the public, because this study was employed in an opt-out method and consent was not obtained directly from all patients. But the datasets are available from the corresponding author on reasonable request.

\section{Competing interests}

The authors have no conflicts of interest to declare.

\section{Funding Sources}

The authors declare that they have no competing interests

\section{Authors' Contributions}

TT is the first author, while TH takes responsibility for the paper as a whole. TT, AN, and TH conceived the study and designed the trial. All authors conducted data collection. TT, NM, TY, KA, HO, AN, and TH conducted data analysis and interpretation. All authors contributed substantially to its revision. 


\section{Acknowledgements}

The authors thank the contribution of our colleagues and their institutions. We would also like to thank Editage (www.editage.com) for the English language editing.

\section{References}

1. Marston A, Pheils MT, Thomas ML, Morson BC. Ischaemic colitis. Gut 7:1-15, 1966.

2. Hreinsson JP, Gumundsson S, Kalaitzakis E, Björnsson ES. Lower gastrointestinal bleeding: incidence, etiology, and outcomes in a population-based setting. Eur J Gastroenterol Hepatol 25:3743, 2013.

3. Chavalitdhamrong D, Jensen DM, Kovacs TOG, et al. Ischemic colitis as a cause of severe hematochezia: risk factors and outcomes compared with other colon diagnoses. Gastrointest Endosc 74:852-857, 2011.

4. Arroja B, Cremers I, Ramos R, et al. Acute lower gastrointestinal bleeding management in Portugal: a multicentric prospective 1-year survey. Eur J Gastroenterol Hepatol 23:317-322, 2011.

5. Higgins PDR, Davis KJ, Laine L. Systematic review: the epidemiology of ischaemic colitis. Aliment Pharmacol Ther 19:729-738, 2004.

6. Walker AM, Bohn RL, Cali C, Cook SF, Ajene AN, Sands BE.Risk factors for colon ischemia. Am J Gastroenterol 99:1333-1337, 2004.

7. Scharff JR, Longo WE, Vartanian SM, Jacobs DL, Bahadursingh AN, Kaminski DL. Ischemic colitis: spectrum of disease and outcome. Surgery 134:624-630, 2003.

8. Cubiella Fernández J, Núñez Calvo L, González Vázquez E, et al. Risk factors associated with the development of ischemic colitis. World J Gastroenterol 16:4564-4569, 2010.

9. Medina C, Vilaseca J, Videla S, Fabra R, Armengol-Miro JR, Malagelada JR.Outcome of patients with ischemic colitis: review of fifty-three cases. Dis Colon Rectum 47:180-184, 2004.

10. Longo WE, Ballantyne GH, Gusberg RJ. Ischemic colitis: patterns and prognosis. Dis Colon Rectum 35:726-730, 1992.

11. Arnott ID, Ghosh S, Ferguson A. The spectrum of ischaemic colitis. Eur J Gastroenterol Hepatol 11:295-303, 1999.

12. Guttormson NL, Bubrick MP. Mortality from ischemic colitis. Dis Colon Rectum 32:469-472, 1989.

13. Acosta S, Ogren M, Sternby NH, Bergqvist D, Björck M. Fatal colonic ischemia: a population-based study. Scand J Gastroenterol 41:1312-1319, 2006.

14. Green BT, Tendler DA. Ischemic colitis: a clinical review. South Med J 98:217-222, 2005.

15. Chang L, Kahler KH, Sarawate C, Quimbo R, Kralstein J.Assessment of potential risk factors associated with ischaemic colitis. Neurogastroenterol Motil 20:36-42, 2008.

16. Brandt LJ, Feuerstadt P, Longstreth GF, Boley SJ; American College of Gastroenterology. ACG clinical guideline: epidemiology, risk factors, patterns of presentation, diagnosis, and management of colon 
ischemia (Cl). Am J Gastroenterol 110:18-45, 2015.

17. Huerta C, Rivero E, Montoro MA, García-Rodriguez LA. Risk factors for intestinal ischaemia among patients registered in a UK primary care database: a nested case-control study. Aliment Pharmacol Ther 33:969-978, 2011.

18. Sherid M, Sifuentes H, Samo S, et al. Ischemic colitis: a forgotten entity. Results of a retrospective study in 118 patients. J Dig Dis 15:606-613, 2014.

19. Sherid M, Ehrenpreis ED. Types of colitis based on histology. Dis Mon 57:457-489, 2011.

20. Deana DG, Dean PJ. Reversible ischemic colitis in young women. Association with oral contraceptive use. Am J Surg Pathol 19:454-462, 1995.

21. Balthazar EJ, Yen BC, Gordon RB. Ischemic colitis: CT evaluation of 54 cases. Radiology 211:381388, 1999.

22. Cervera R, Espinosa G, Cordero A, et al. Intestinal involvement secondary to the antiphospholipid syndrome (APS): clinical and immunologic characteristics of 97 patients: comparison of classic and catastrophic APS. Semin Arthritis Rheum 36:287-296, 2007.

23. Preventza OA, Lazarides K, Sawyer MD. Ischemic colitis in young adults: a single-institution experience. J Gastrointest Surg 5:388-392, 2001.

24. Sherid M, Sifuentes H, Samo S, et al. Risk factors of recurrent ischemic colitis: a multicenter retrospective study. Korean J Gastroenterol 63:283-291, 2014.

25. Longstreth GF, Yao JF. Epidemiology, clinical features, high-risk factors, and outcome of acute large bowel ischemia. Clin Gastroenterol Hepatol 7:1075-1023, 2009.

26. Glauser PM, Wermuth P, Cathomas G, Kuhnt E, Käser SA, Maurer CA.Ischemic colitis: clinical presentation, localization in relation to risk factors, and long-term results. World J Surg 35:25492554, 2011.

27. Huguier M, Barrier A, Boelle PY, Houry S, Lacaine F.Ischemic colitis. Am J Surg 192:679-684, 2006.

28. Dasso JF, Obiakor H, Bach H, Anderson AO, Mage RG. A morphological and immunohistological study of the human and rabbit appendix for comparison with the avian bursa. Dev Comp Immunol 24:797-814, 2000.

29. Fujihashi K, McGhee JR, Lue $C$, et al. Human appendix B cells naturally express receptors for and respond to interleukin 6 with selective IgA1 and IgA2 synthesis. J Clin Invest 88:248-252, 1991.

30. Masahata K, Umemoto E, Kayama H, et al. Generation of colonic IgA-secreting cells in the caecal patch. Nat Commun 5:3704, 2014.

31. Kruglov AA, Grivennikov SI, Kuprash DV, et al. Nonredundant function of soluble LTa3 produced by innate lymphoid cells in intestinal homeostasis. Science 342:1243-1246, 2013.

32. Sahami S, Kooij IA, Meijer SL, Van den Brink GR, Buskens CJ, Te Velde AA. The link between the appendix and ulcerative colitis: clinical relevance and potential immunological mechanisms. Am J Gastroenterol 111:163-169, 2016. 
33. Koutroubakis IE, Vlachonikolis IG. Appendectomy and the development of ulcerative colitis: results of a metaanalysis of published case-control studies. Am J Gastroenterol 95:171-176, 2000.

34. Radford-Smith GL, Edwards JE, Purdie DM, et al. Protective role of appendicectomy on onset and severity of ulcerative colitis and Crohn's disease. Gut 51:808-813, 2002.

35. Yadav S, Dave M, Varayil JE, et al. A population-based study of incidence, risk factors, clinical spectrum, and outcomes of ischemic colitis. Clin Gastroenterol Hepatol 13:731-738.e1-e6, 2015.

\section{Figures}

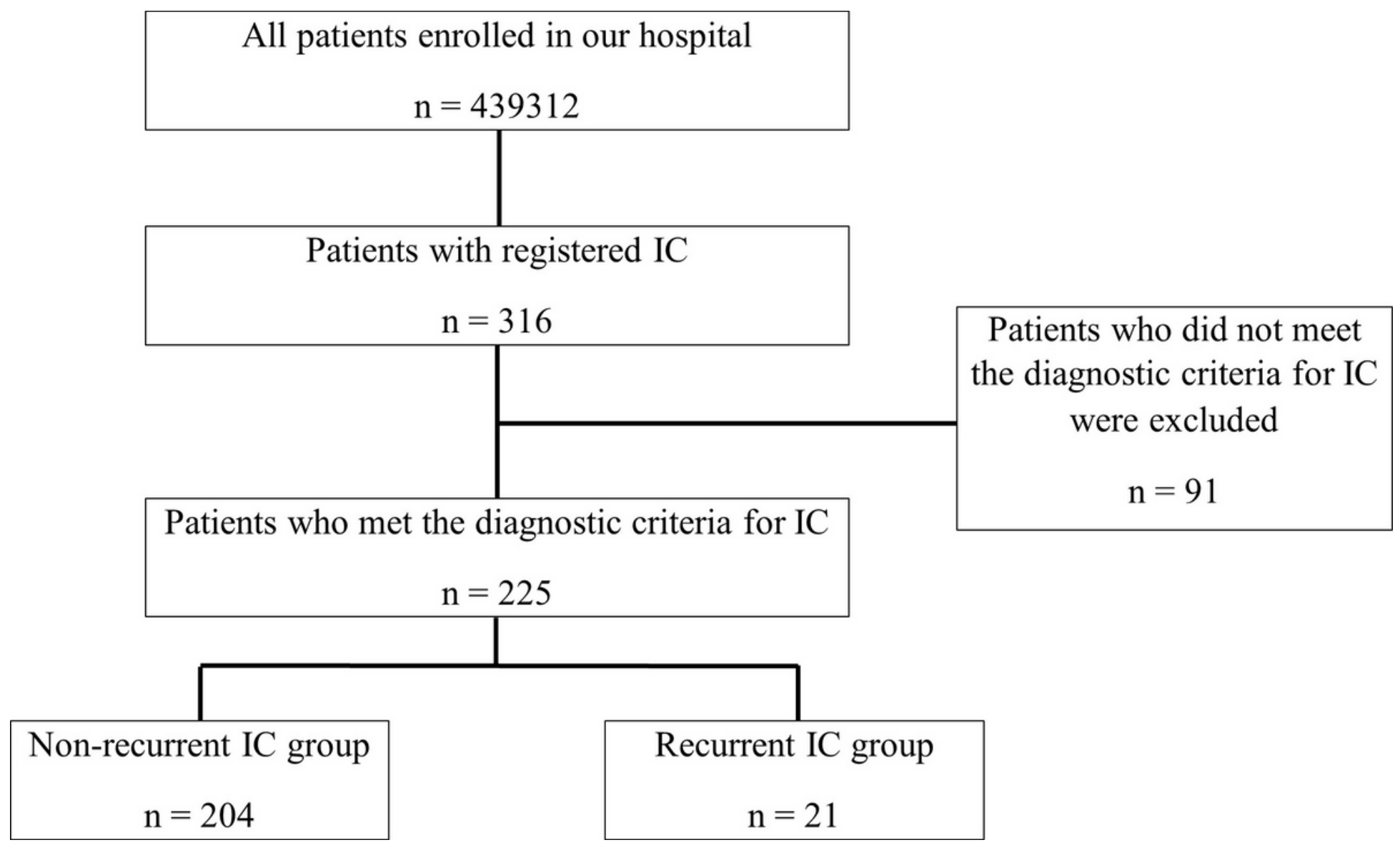

Figure 1

Patient enrollment and study flow

IC, ischemic colitis 


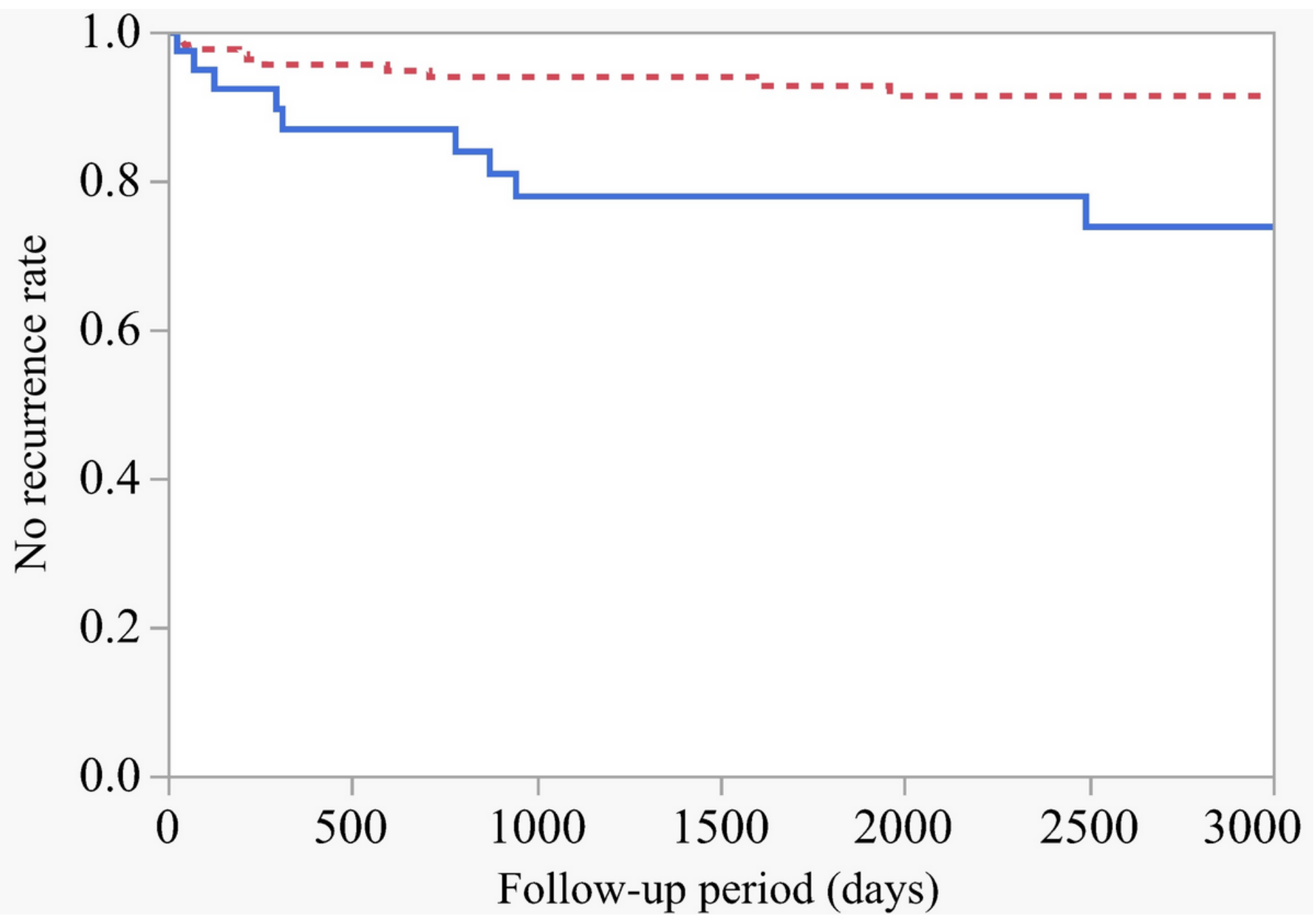

Figure 2

Time to ischemic colitis recurrence in patients with and without a history of appendectomy

Dotted line, without previous appendectomy; solid line, with previous appendectomy 


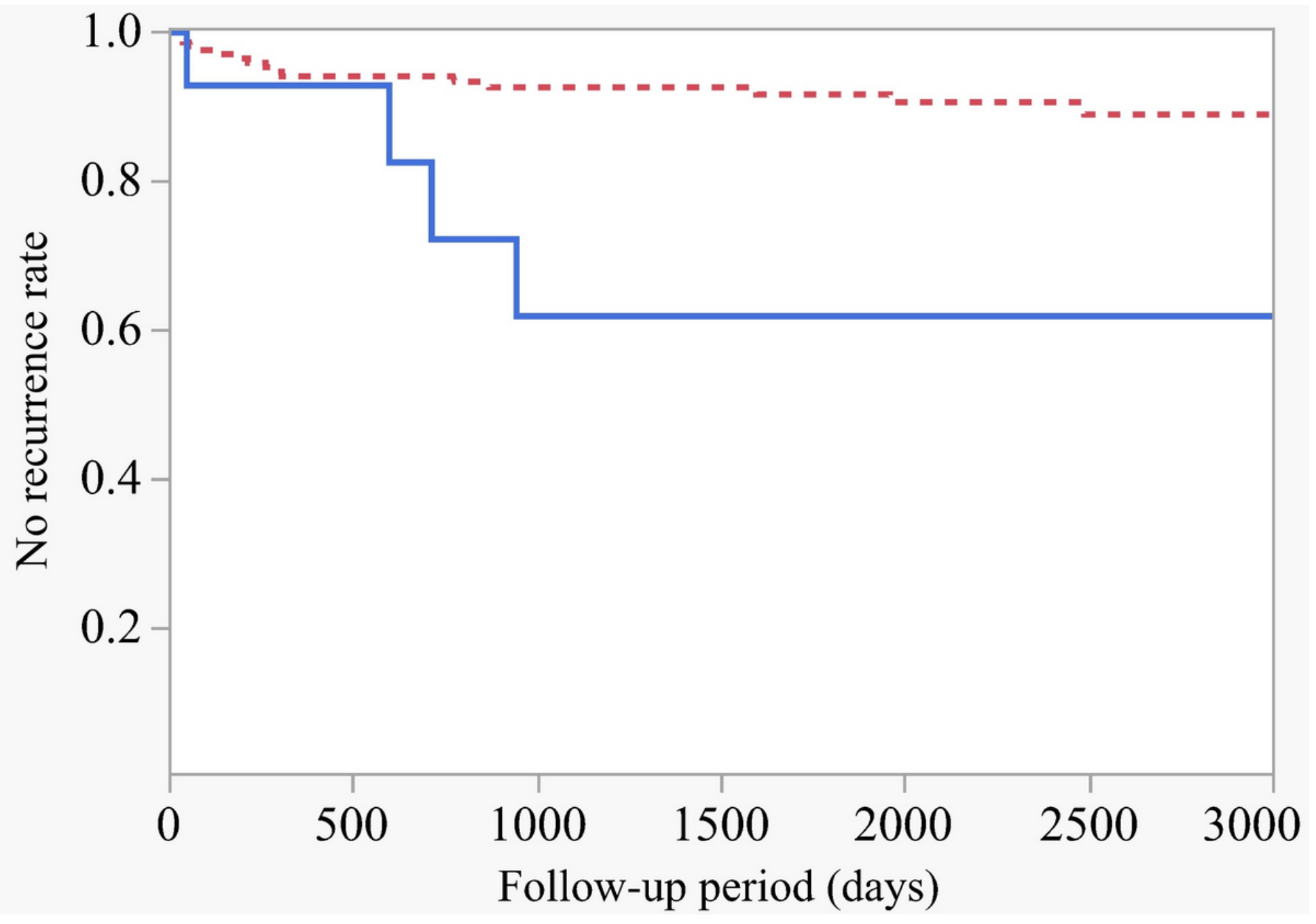

Figure 3

Time to ischemic colitis recurrence in patients with and without a history of cerebral infarction Dotted line, without cerebral infarction; solid line, with cerebral infarction 


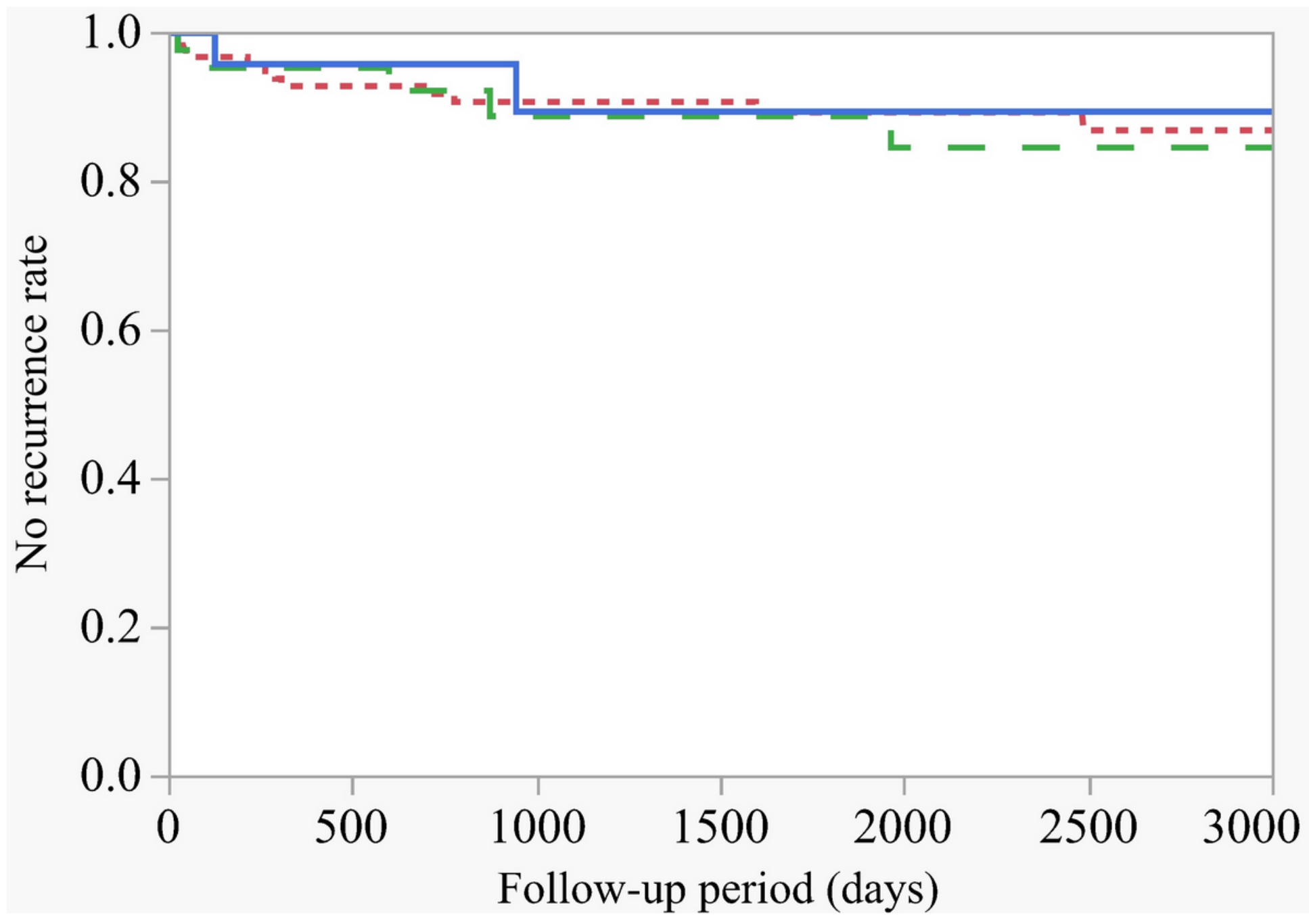

Figure 4

Time to ischemic colitis recurrence in patients with and without a history of smoking Dotted line, non-smoker; chain line, past smoker; solid line, current smoker 


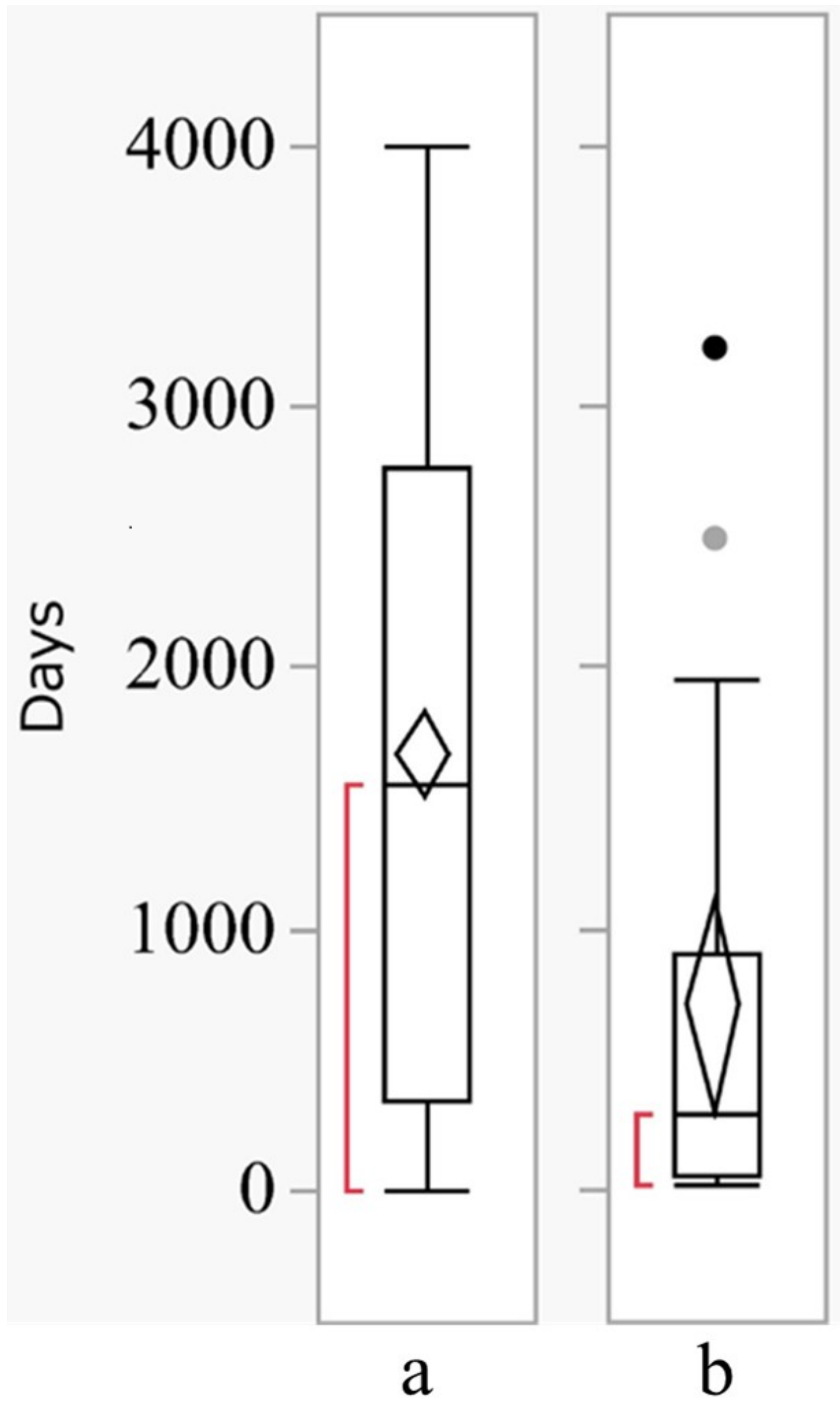

Figure 5

Follow-up period and time to recurrence of ischemic colitis

(a) Follow-up period. (b) Time to ischemic colitis recurrence. 
The error bars indicate the maximum and minimum values of the data in the range of 1.5 times from the median to the quartiles (but the lower limit is zero). The diamonds represent the confidence intervals. 